\title{
THE COMPARABILITY GRAPH OF A TREE
}

\author{
E. S. WOLK ${ }^{1}$
}

1. Introduction. By an unoriented graph we mean a pair $(G, R)$, where $G$ is a set (finite or infinite) and $R$ is an irreflexive and symmetric relation defined on $G$. If $R$ is a relation on $G$ (i.e., a subset of $G \times G)$, we write $x R y$ if and only if $(x, y) \in R$. If $(x, y) \in G \times G$ and $(x, y) \notin R$, then we write $x \tilde{R} y$. An oriented graph is a pair $(G, S)$, where $G$ is a set and $S$ is a relation on $G$ which is irreflexive and antisymmetric (i.e., $x S y$ and $y S x$ do not both hold for any $x \in G$, $y \in G)$. An oriented graph $(G, S)$ is transitively oriented if and only if, for all $x, y, z$ in $G, x S y$ and $y S z$ imply $x S z$. A transitively oriented graph is a partially ordered set, and in this case $S$ is called a partial order of $G$. $(H, S)$ is a subgraph of $(G, R)$ if and only if (i) $H \subseteq G$ and (ii) $x S y$ if and only if $x R y$, for all $x, y \in H$. For brevity we may sometimes suppress mention of the relations $R$ or $S$, and refer only to a graph $G$ or a subgraph $H$. Whenever possible we may form an intuitive picture of a graph $(G, R)$ by imagining the elements of $G$ to be points in the plane, and interpreting $x R y$ to mean that $x$ and $y$ are connected by a line segment (directed or undirected according as $(G, R)$ is oriented or not).

The general problem which we propose to investigate may be described as follows. Let $(G, R)$ be an unoriented graph. We say that a relation $T$ on $G$ is a transitive orientation of $(G, R)$ if and only if (i) $(G, T)$ is a transitively oriented graph, and (ii) $x R y$ if and only if $x T y$ or $y T x$, for all $x, y \in G$. Now we may ask the question: what are necessary and sufficient conditions on an unoriented graph $(G, R)$ for $(G, R)$ to possess a transitive orientation?

The problem may also be stated in a slightly different form. Let $(G,<)$ be a partially ordered set. We may construct from $(G,<)$ an unoriented graph $(G, R)$ by defining $x R y$ if and only if $x<y$ or $y<x$; i.e., $x R y$ if and only if $x$ and $y$ are comparable elements of $G$ with respect to the partial order $<$. We shall call $(G, R)$ the comparability graph of $(G,<)$. Then our question is the following. Given an unoriented graph $(G, R)$, under what conditions is $(G, R)$ the comparability graph of a partially ordered set?

It is easy to discover a number of necessary conditions. For example, if $(G, R)$ contains any closed polygon with an odd number of

Received by the editors August 3, 1961.

1 This research was supported by a National Science Foundation grant (No. G16314). 
sides (greater than 3 ), then $(G, R)$ must also contain a diagonal of this polygon. Also, there are certain six-element graphs which possess no transitive orientation. For example, let $G=\{a, b, c, d, e, f\}$ and let

$$
\begin{aligned}
& R_{1}=\{(a, b),(b, c),(a, c),(a, d),(b, e),(c, f)\}, \\
& R_{2}=\{(a, b),(b, c),(a, c),(a, d),(b, d),(b, e),(c, e),(a, f),(c, f)\} .
\end{aligned}
$$

The reader may easily verify that neither $\left(G, R_{1}\right)$ nor $\left(G, R_{2}\right)$ admit a transitive orientation. Thus, the comparability graph of a partially ordered set may contain neither $\left(G, R_{1}\right)$ nor $\left(G, R_{2}\right)$ as a subgraph.

The problem we have formulated appears to be very difficult, in its most general form, and hence it seems natural to make a first attack by restricting the class of partially ordered sets under discussion. Let us say that a partially ordered set $(G,<)$ is a tree if and only if whenever $x$ and $y$ are incomparable elements of $G$ there exists no $z \in G$ with $x<z$ and $y<z$. Our purpose in this paper is to characterize the comparability graph of a tree; i.e., we obtain a simple necessary and sufficient condition that an unoriented graph $(G, R)$ possess a transitive orientation $T$ such that $(G, T)$ is a tree. We intend, in subsequent work, to attempt to extend our methods to larger classes of partially ordered sets.

2. Main result. Throughout this paper set inclusion will be denoted by $\subseteq$, and $\subseteq$ will be reserved for proper inclusion. $A-B$ denotes the relative complement of the set $B$ with respect to the set $A$. If $R$ is a relation on a set $M$ and $A \subseteq M$, we define $R / A=R \cap(A \times A)$.

Some further definitions relating to unoriented graphs will be necessary. A subgraph $H$ of an unoriented graph $(G, R)$ will be called complete if and only if $x \in H$ and $y \in H$ imply $x R y$. A subgraph $H$ of $(G, R)$ is empty if and only if $x \in H$ and $y \in H$ imply $x \widetilde{R} y$. $H$ is a maximal complete subgraph of $G$ if and only if $H$ is complete and there exists no complete subgraph $K$ of $G$ with $H \subset K$. It is an immediate consequence of Zorn's Lemma that every complete subgraph of a graph $G$ is contained in a maximal complete subgraph of $G$.

The following definition plays a central role in our discussion. A related but weaker condition has recently been considered by Hajnal and Surányi [1].

Definition. An unoriented graph $(G, R)$ has the diagonal property if and only if whenever $x_{1}, x_{2}, x_{3}$, and $x_{4}$ are elements of $G$ with $x_{1} R x_{2} R x_{3} R x_{4}$, then we also have $x_{1} R x_{3}$ or $x_{2} R x_{4}$.

The reader may easily verify that the diagonal property is equivalent to the following condition: whenever $\left\{x_{0}, x_{1}, \cdots, x_{k}\right\}$ is a finite sequence of elements of $G$ with $x_{0} R x_{1} R \cdots R x_{k}$, there exists 
an $i$ such that $1 \leqq i \leqq k-1$ and $x_{0} R x_{i} R x_{k}$.

We now state our main theorem. No restriction is placed on the cardinal number of $G$.

TheOREM. An unoriented graph $(G, R)$ is the comparability graph of a tree if and only if $(G, R)$ has the diagonal property.

We first prove the necessity of the diagonal property. Suppose that $(G, R)$ is the comparability graph of a tree $(G,<)$. Then $x R y$ means that $x<y$ or $y<x$. Suppose that $(G, R)$ does not have the diagonal property. Then there exist elements $a, b, c$, and $d$ in $G$ such that $a R b R c R d$, but $a \tilde{R} c$ and $b \tilde{R} d$. Let us consider two cases.

Case 1. Suppose that $a<b$. Then, since $c$ is comparable with $b$ but not with $a$, we must have $c<b$. But $a<b$ and $c<b$ contradicts the condition that $(G,<)$ is a tree.

Case 2. Suppose that $b<a$. Then we must have $b<c$, since $c$ is not comparable with $a$. Likewise we must have $d<c$. But $b<c$ and $d<c$ is again a contradiction.

The proof of the sufficiency of the diagonal property is less simple. Given an unoriented graph $(G, R)$ with the diagonal property, we are of course required to produce a transitive orientation $T$ of $(G, R)$ such that the oriented graph $(G, T)$ is a tree. The construction of the relation $T$ will be broken down into a sequence of lemmas. In each of the following lemmas $(G, R)$ is assumed to be an unoriented graph with the diagonal property.

Lemma 1. Let $N$ be a maximal complete subgraph of $(G, R)$. Let $a$ be any family of maximal complete subgraphs of $(G, R)$ with $N \notin Q$. Then the family $\{M \cap N: M \in a\}$ is a nest of sets.

Proof. If the lemma is false, then there exist $M_{1} \in a, M_{2} \in a$ such that $M_{1} \cap N$ and $M_{2} \cap N$ are incomparable sets. Suppose that $x_{1} \in\left(M_{1}-M_{2}\right) \cap N, x_{2} \in\left(M_{2}-M_{1}\right) \cap N$. We have $x_{1} R x_{2}$, since $x_{1}, x_{2}$ $\in N$. Also, by maximality of $M_{2}, x_{1} \notin M_{2}$ implies that there exists $q \in M_{2}$ with $x_{1} \widetilde{R} q$. Likewise, $x_{2} \notin M_{1}$ implies that there exists $p \in M_{1}$ with $x_{2} \tilde{R} p$. Then $p R x_{1} R x_{2} R q$ but $p \tilde{R} x_{2}$ and $x_{1} \tilde{R} q$, contradicting the diagonal property.

For any $m \in G$, let us define $G_{m}=\{x \in G: x R m$ or $x=m\}$.

LeMma 2. Let $C$ be any complete subgraph of $(G, R)$. Then the family $\left\{G_{m}: m \in C\right\}$ is a nest of sets.

Proof. Suppose that $G_{m}$ and $G_{n}$ are incomparable sets for some $m, n \in C$. Let $x \in G_{m}-G_{n}, y \in G_{n}-G_{m}$. Then $x R m R n R y$, but $x \widetilde{R} n$ and $m \tilde{R} y$, a contradiction. 
The construction of the transitive orientation $T$ for the graph $(G, R)$ will be accomplished by an inductive procedure. By a linearly ordered set we mean a partially ordered set in which each two distinct elements are comparable. Let $\mathfrak{T}$ denote the set of all maximal complete subgraphs of $(G, R)$. Roughly speaking, we shall define inductively a linear order on each $M \in \mathscr{N}$ in such a way that, if $R_{M}$ and $R_{N}$ are the linear orders on $M$ and $N$ respectively, then $R_{M}$ and $R_{N}$ agree on $M \cap N$. The transitive orientation $T$ will then be defined as the union of all the $R_{M}$ 's. The members of the family $\mathfrak{N}$ will of course be the maximal chains of the partially ordered set $(G, T)$.

First we give two more definitions.

Definition. Let $M \in \mathfrak{T}$. If $R_{M}$ is a linear order on $M$, we say that $R_{M}$ is admissible for $M$ if and only if $m \in M, n \in M$, and $G_{n} \subset G_{m}$ imply $m R_{M} n$.

Definition. Let $(A,<)$ be a linearly ordered set and let $B \subseteq A$. We say that $B$ is an initial section of $A$ if and only if $b \in B, x \in A$, and $x<b$ imply $x \in B$.

LEMma 3. Each $M \in \mathfrak{T}$ possesses an admissible linear ordering $R_{M}$.

Proof. Let $M \in \mathscr{T}$. For $m, n \in M$, let us define $m \sigma_{M} n$ if and only if $G_{m}=G_{n}$. The relation $\sigma_{M}$ is an equivalence relation on $M$. Let $\mathcal{E}$ denote the set of all equivalence classes with respect to $\sigma_{M}$. For any $E \in \mathcal{E}$, let $R_{E}$ be an arbitrary linear ordering of $E$. Then we define

$$
R_{M}=\bigcup\left\{R_{E}: E \in \varepsilon\right\} \cup\left\{(m, n) \in M \times M: G_{n} \subset G_{m}\right\} .
$$

Since the family $\left\{G_{m}: m \in M\right\}$ is a nest, by Lemma 2 , it follows immediately that $R_{M}$ is an admissible linear ordering of $M$.

Now let $\mathfrak{T}$ be well-ordered, so that $\mathscr{T}=\left\{M_{\alpha}: \alpha \in W\right\}$, where $W$ is some ordinal $\{0,1, \cdots, \alpha, \cdots\}$. For each $\alpha \in W$, we shall define by transfinite induction a relation $R_{\alpha}$ satisfying

(1) $R_{\alpha}$ is an admissible linear ordering of $M_{\alpha}$,

(2) for each $\beta<\alpha$, we have $R_{\alpha} /\left(M_{\alpha} \cap M_{\beta}\right)=R_{\beta} /\left(M_{\alpha} \cap M_{\beta}\right)$,

(3) for each $\beta<\alpha, M_{\alpha} \cap M_{\beta}$ is an initial section of both $M_{\alpha}$ and $M_{\beta}$ in the linear orders $R_{\alpha}$ and $R_{\beta}$ respectively.

Choose $R_{0}$ as any admissible linear order on $M_{0}$. Let $\alpha \in W$, and assume that $R_{\beta}$ has been defined satisfying (1), (2), and (3) for all $\beta<\alpha$. We shall show how to define $R_{\alpha}$.

The family $\left\{M_{\alpha} \cap M_{\beta}: \beta<\alpha\right\}$ is a nest of sets, by Lemma 1 . Let $Z=\bigcup\left\{M_{\alpha} \cap M_{\beta}: \beta<\alpha\right\}$, and let $S=\bigcup\left\{R_{\beta} /\left(M_{\alpha} \cap M_{\beta}\right): \beta<\alpha\right\}$.

LemMa 4. $S$ is a linear order on $Z$.

Proof. We first check that $S$ is transitive. Suppose that $x S y$ 
and $y S z$. Then there exist $\gamma<\alpha$ and $\delta<\alpha$ such that $(x, y)$ $\in R_{\gamma} /\left(M_{\alpha} \cap M_{\gamma}\right)$ and $(y, z) \in R_{\delta} /\left(M_{\alpha} \cap M_{\delta}\right)$. Since the family $\left\{M_{\alpha} \cap M_{\beta}: \beta<\alpha\right\}$ is a nest, the sets $M_{\alpha} \cap M_{\gamma}$ and $M_{\alpha} \cap M_{\delta}$ are comparable. Assume that $M_{\alpha} \cap M_{\gamma} \subseteq M_{\alpha} \cap M_{\delta}$. Then $x, y$, and $z$ are all elements of $M_{\alpha} \cap M_{\delta}$, and $R_{\delta}$ is a linear order on $M_{\delta}$ by our inductive hypothesis. Hence $(x, z) \in R_{\delta} /\left(M_{\alpha} \cap M_{\delta}\right)$, and thus $x S z$. The proof that $x S y$ or $y S x$ for all $x, y \in Z$, with $x \neq y$, is very similar and may be left to the reader.

Now let $R_{M_{\alpha}}$ be any admissible linear order on $M_{\alpha}$. We define

$$
R_{\alpha}=S \cup R_{M_{\alpha}} /\left(M_{\alpha}-Z\right) \cup\left\{(x, y): x \in Z, y \in M_{\alpha}-Z\right\} \text {. }
$$

Lemma 5. $R_{\alpha}$ satisfies (1), (2), and (3).

Proof. (1) It is clear that $R_{\alpha}$ is a linear order on $M_{\alpha}$. Let us show that $R_{\alpha}$ is admissible. Suppose that $x, y \in M_{\alpha}$ and $G_{y} \subset G_{x}$. We must consider several cases.

Case I. $x \in Z, y \in Z$. In this case we have $x, y \in M_{\alpha} \cap M_{\delta}$ for some $\delta<\alpha$. By our inductive hypothesis $R_{\delta}$ is an admissible linear order on $M_{\delta}$. Hence $(x, y) \in R_{\delta} /\left(M_{\alpha} \cap M_{\delta}\right) \subseteq S$. Hence $(x, y) \in R_{\alpha}$.

Case II. $x \in M_{\alpha}-Z, y \in M_{\alpha}-Z$. The result in this case follows immediately by the admissibility of $R_{M_{\alpha}}$.

Case III. $x \in M_{\alpha}-Z, y \in Z$. We show that this case cannot occur. For suppose that $y \in M_{\alpha} \cap M_{\beta}$ for some $\beta<\alpha$. Then $x \notin M_{\beta}$. Hence, by maximality of $M_{\beta}$, there exists $p \in M_{\beta}$ with $x \tilde{R} p$. Hence $p \notin G_{3}$ but $p \in G_{y}$, contradicting $G_{y} \subset G_{x}$.

In the remaining case when $x \in Z, y \in M_{\alpha}-Z$, we have at once that $(x, y) \in R_{\alpha}$. Hence the proof of (1) is complete.

(2) Let $\beta<\alpha$. Then $R_{\alpha} /\left(M_{\alpha} \cap M_{\beta}\right)=S /\left(M_{\alpha} \cap M_{\beta}\right)$ by definition of $R_{\alpha}$. But by definition of $S$ we have $S /\left(M_{\alpha} \cap M_{\beta}\right)=R_{\beta} /\left(M_{\alpha} \cap M_{\beta}\right)$. This proves (2).

(3) Let $\beta<\alpha$. Let $x \in M_{\alpha} \cap M_{\beta}, y \in M_{\beta}-M_{\alpha}$, and $z \in M_{\alpha}-M_{\beta}$. We must prove that $x R_{\beta} y$ and $x R_{\alpha} z$. Since $y \notin M_{\alpha}$, by maximality of $M_{\alpha}$ there exists $p \in M_{\alpha}$ with $y \tilde{R} p$. Hence $p \notin G_{y}$, but $p \in G_{x}$. Since the family $\left\{G_{m}: m \in M_{\beta}\right\}$ is a nest, we have $G_{\nu} \subset G_{x}$. Hence $x R_{\beta} y$, since $R_{\beta}$ is an admissible linear order on $M_{\beta}$. In a similar way we show that $z \notin M_{\beta}$ implies $G_{z} \subset G_{x}$, and hence $x R_{\alpha} z$.

We now define $T=\bigcup\left\{R_{\alpha}: \alpha \in W\right\}$.

Lemma 6. For all $x, y \in G$, we have $x R$ if and only if $x T y$ or $y T x$.

Proof. $x T y$ implies $x R_{\alpha} y$ for some $\alpha$. Thus $x$ and $y$ are elements of the complete subgraph $M_{\alpha}$ and hence $x R y$. Conversely, suppose that $x R y$. Then there exists a maximal complete subgraph $M_{\alpha}$ such 
that $x, y \in M_{\alpha}$. Then $x R_{\alpha} y$ or $y R_{\alpha} x$, since $R_{\alpha}$ is a linear order on $M_{\alpha}$. Hence $x T y$ or $y T x$.

To conclude the proof of our theorem, we shall now show that $(G, T)$ is a tree. We first prove that $(G, T)$ is a partially ordered set. $T$ is irreflexive, since all $R_{\alpha}$ have this property, so it is necessary only to check the transitivity of $T$. Suppose that $x T y$ and $y T z$ for some $x, y$, and $z$ in $G$. We shall show that the set $\{x, y, z\}$ forms a complete subgraph of $(G, R)$. By Lemma 6 we know that $x R y$ and $y R z$, so we need only to show that $x R z$. First note that if $G_{x}=G_{y}$, then it follows at once that $x R z$, since $z \in G_{y}$. So assume that $G_{x} \neq G_{y}$. In this case, since $x R_{\alpha} y$ for some $\alpha$, and the linear order $R_{\alpha}$ is admissible, we must have $G_{y} \subset G_{x}$. Hence there exists $q \in G$ such that $q R x$ and $q \widetilde{R} y$. Then $q R x R y R z$ but $q \tilde{R} y$. By the diagonal property we have $x R z$. Therefore $\{x, y, z\}$ is a complete subgraph of $(G, R)$. Hence there exists $\gamma \in W$ such that $\{x, y, z\} \subseteq M_{\gamma}$. But $x T y$ implies $x R_{\gamma} y$, and $y T z$ implies $y R_{\gamma} z$. Since $R_{\gamma}$ is transitive on $M_{\gamma}$, we have $x R_{\gamma} z$, and hence $x T z$.

Now to show that $(G, T)$ is a tree, let $x$ and $y$ be elements of $G$ with $x \tilde{T} y$ and $y \tilde{T} x$, and suppose that there exists $z \in G$ with $x T z$ and $y T z$. Then there exist $\alpha, \beta \in W$ with $x, z \in M_{\alpha}$ and $y, z \in M_{\beta}$. Since $x \tilde{R} y$, we have $x \notin M_{\beta}, y \notin M_{\alpha}$. Also, by the construction of $T$, we have $x R_{\alpha} z$ and $y R_{\beta} z$. But, since $z \in M_{\alpha} \cap M_{\beta}$, this means that $M_{\alpha} \cap M_{\beta}$ is not an initial section of $M_{\alpha}$ or of $M_{\beta}$, contradicting condition (3). Hence $(G, T)$ is a tree, and the proof of the theorem is complete.

3. Some remarks on Souslin's Problem. If $G$ is a partially ordered set and $H \subseteq G$, we say that $H$ is totally unordered if and only if all pairs of distinct elements of $H$ are incomparable. E. W. Miller [2] has shown that the well-known Problem of Souslin may be stated in the following equivalent form. If $(G,<)$ is a tree in which all chains and all totally unordered subsets are countable, does it follow that $G$ is countable? Since we have shown that the diagonal property characterizes the comparability graph of a tree, we are now able to state Souslin's Problem in a "graph-theoretic" form as follows. If $G$ is an unoriented graph with the diagonal property in which all complete subgraphs and all empty subgraphs are countable, then must $G$ be countable? It should be noted that the affirmation of this proposition is a weaker statement than an open question relating to Souslin's Problem recently discussed by Hajnal and Surányi [1].

We may also give another formulation to Souslin's Problem in the following way. Let us call a partially ordered set $(G,<)$ a pseudo-tree if and only if the comparability graph of $(G,<)$ has the diagonal 
property. A simple example of a pseudo-tree which is not a tree is the partially ordered set consisting of a least element 0 , a greatest element $I$, and a pair of incomparable elements $x, y$, with $0<x<I, 0<y<I$. The reader may easily verify that a partially ordered set $(G,<)$ is a pseudo-tree if and only if it satisfies the following condition: whenever $a, b, c, d$ are distinct elements of $G$ such that $a$ and $b$ are incomparable, $c<a, c<b$, and $d<b$, then $d$ is comparable with $c$. Now, according to our theorem of the preceding section, given any pseudotree $P$, there exists a mapping of $P$ onto a tree which is $1: 1$ and which preserves the comparability relation. Thus we may state Souslin's Problem as follows. Does there exist an uncountable pseudo-tree in which (i) every chain is countable and (ii) every totally unordered subset is countable?

It is important to point out that there exist uncountable partially ordered sets satisfying conditions (i) and (ii) above. An example of such a partially ordered set is due, in essence, to Sierpinski [3]. Let $G$ denote the set of all ordinals preceding the first uncountable ordinal, and let $E_{1}$ be the real numbers. Let $<$ denote the usual ordering of both $G$ and $E_{1}$. Let $f$ be a $1: 1$ mapping of $G$ into $E_{1}$. For $x, y \in G$, we define $x T y$ if and only if $x<y$ and $f(x)<f(y) .(G, T)$ is an uncountable partially ordered set, and Sierpinski's results [3] imply that $(G, T)$ satisfies conditions (i) and (ii).

\section{REFERENCES}

1. A. Hajnal and J. Surányi, Über die Auflösung von Graphen in vollständige Teilgraphen, Ann. Univ. Sci. Budapest, Eötvös, Sect. Math. 1 (1958), 113-121.

2. E. W. Miller, A note on Souslin's Problem, Amer. J. Math. 65 (1943), 673-678.

3. W. Sierpinski, Sur un probleme de la theorie des relations, Ann. Scuola Norm. Sup. Pisa 2 (1933), 285-287.

UNiversity OF CONNECTICUT 